\title{
REHABILITACIÓN NEUROPSICOLÓGICA EN PERSONAS CON SÍNDROME DE PRADER-WILLI
}

\section{Neuropsychological rehabilitation in people with Prader-Willi syndrome}

\author{
Karol TAPIA DE MOYA \\ Universidad Autónoma de Madrid. Oficina de Acción Solidaria y Cooperación. C/ Einstein, 7. \\ Campus de Cantoblanco. Edificio Plaza Mayor. Madrid \\ karol.tapia@inv.uam.es
}

Recepción: 2 de octubre de 2015

Fecha de aceptación definitiva: 31 de enero de 2017

Resumen: El síndrome de Prader-Willi (SPW) es un trastorno complejo del neurodesarrollo causado por anomalías genéticas y que presenta un fenotipo neuroconductual característico. El objetivo de este trabajo fue analizar las intervenciones de rehabilitación neuropsicológica en SPW, por lo que se realizó una búsqueda sistemática, incluyéndose un total de 14 artículos con intervenciones en características fenotípicas conductuales, cognitivas o familiares. Los estudios fueron analizados según el enfoque de la intervención, modelos, procedimientos específicos y resultados. La mayor parte de los estudios utilizaron un modelo de compensación y casi todos aplicaron técnicas de modificación conductual. Los resultados sugieren que las técnicas que utilizan un reforzamiento diferencial, que incluyen una mayor participación de la persona con SPW y sus familiares en el proceso de intervención, son más exitosas.

Palabras clave: síndrome de Prader-Willi; fenotipo conductual; rehabilitación; neuropsicología.

Abstract: Prader-Willi Syndrome (PWS) is a complex neurodevelopmental disorder caused by genetic anomalies that presents itself with a characteristic neurobehavioral phenotype. The aim of this project was to analyze interventions of neuropsychological 
rehabilitation that exist in PWS and to achieve it, a systematic search was done, resulting in a total of 14 articles with interventions for behavioral, cognitive and familial phenotypical characteristics. The studies were analyzed according to the focus of the intervention, models, specific procedures and results. Most studies used a compensation model and almost all applied behavioral modification techniques. The results suggest that techniques that use differential reinforcement and a more active involvement of the person with PWS and their family during intervention, are more successful.

KEY words: Prader-Willi Syndrome; behavioral phenotype; rehabilitation; neuropsychology.

\section{Introducción}

$\int$ L SíndRome De Prader-Willi (SPW) fue descrito inicialmente por Prader, Labhart y Willi en 1956. Este síndrome, con una incidencia de 1 en 15.000 a 25.000 nacidos vivos, corresponde a un patrón reconocible de hallazgos físicos con anormalidades significativas a nivel cognitivo, neurológico, endocrino y conductual, causadas por una falta de expresión de los genes de una región de impronta del cromosoma 15 heredado paternalmente, siendo las causas más comunes una deleción en la región q11-13 y una disomía uniparental materna (McCandless y Committee on Genetics, 2011).

Se han identificado una serie de criterios principales, secundarios y de apoyo para el diagnóstico del SPW, resumidos en los Criterios Diagnósticos Consensuados (que han sido actualizados y revisados por Gunay-Aygun, Schwartz, Heeger, O’Riordan y Cassidy, 2001). Algunos criterios diagnósticos corresponden al fenotipo neuroconductual, que es definido por Flint y Yule (1994) como un patrón característico a nivel motor, cognitivo, lingüístico y social que muestran pacientes con un trastorno genético o cromosómico específico. Este patrón de características en las personas con SPW se divide clásicamente en dos estadios con subetapas específicas: el primero se evidencia en el recién nacido, quien presenta hipotonía severa, dificultad para chupar y desinterés por los alimentos. El cambio al segundo estadio se va dando de forma gradual y se hace completamente evidente unos años después, caracterizándose por una obsesión por la comida e hiperfagia, y su inicio se da alrededor de los 8 años (McAllister, Whittington y Holland, 2011). Es en este segundo estadio donde se establecen los componentes del fenotipo neuroconductual que más caracterizan a las personas con SPW y que los pueden llevar a presentar problemas de sobrepeso potencialmente mortales, dificultades académicas, dificultades en la socialización, alteraciones conductuales y demás, que si no son tratados pueden llegar a significar un peligro para el funcionamiento (y la misma vida) de la persona con SPW y quienes lo rodean, limitando su participación y autonomía (Whitman y Jackson, 2006).

Debido a la heterogeneidad del SPW en sí mismo y el contexto social y familiar que rodea a la persona, las características del fenotipo neuroconductual podrían presentarse todas juntas o no, y pueden variar en cuanto a su presentación. Como es explicado por el enfoque neuroconstructivista, existe una dinámica ente genes, cerebro 


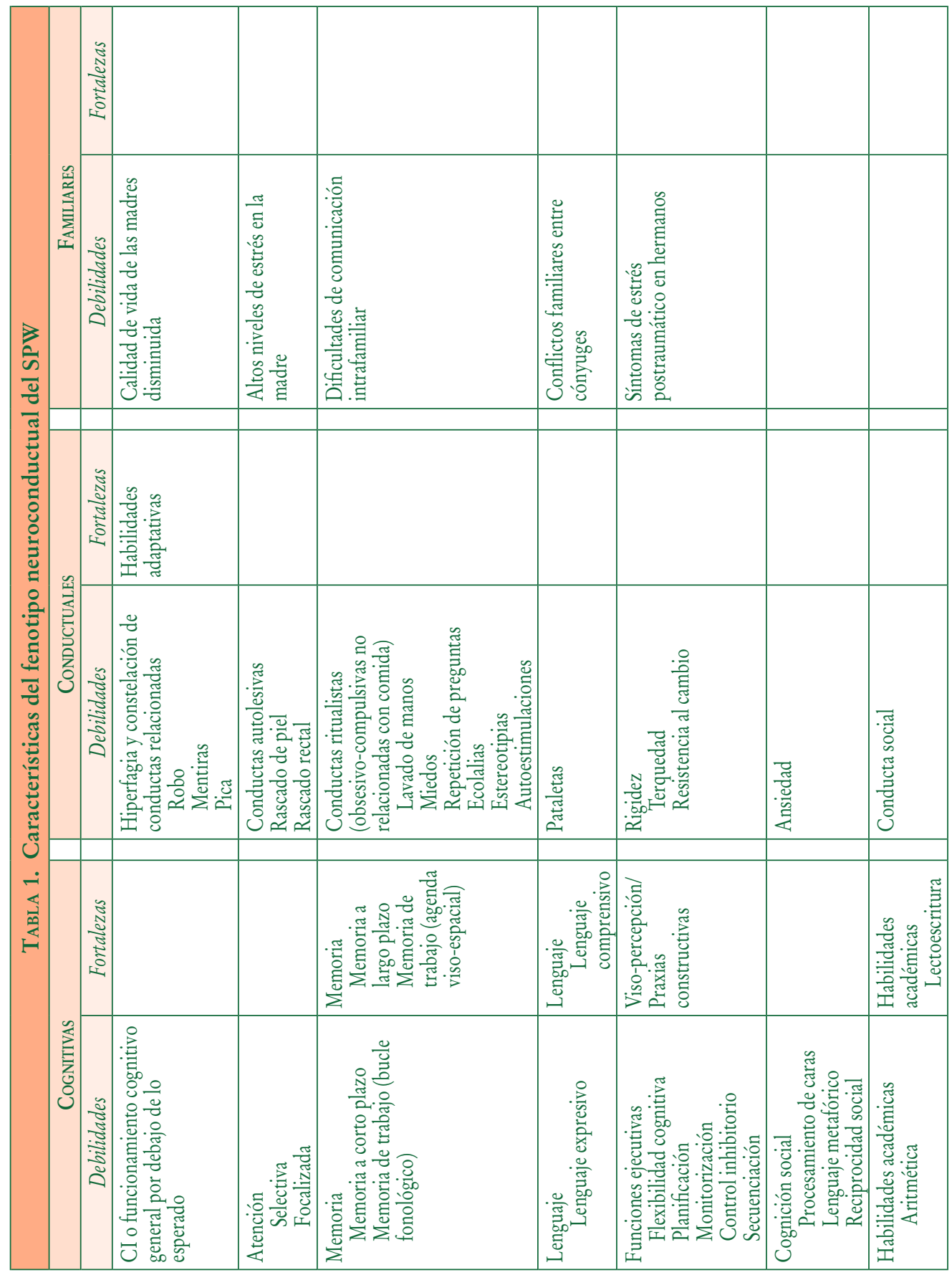

(C) Ediciones Universidad de Salamanca / CC BY-NC-ND

Siglo Cero, vol. 48 (1), n. ${ }^{\circ}$ 261, 2017, enero-marzo, pp. 73-93 
y ambiente que dará forma a las conductas y capacidades que la persona llegará a exhibir (Farran y Karmiloff-Smith, 2011).

Aunque dentro de los criterios actualizados solo se contemplan a nivel conductual la hiperfagia, la obsesión con la comida, las pataletas y los rituales obsesivos-compulsivos, y a nivel cognitivo el retraso en el desarrollo y la discapacidad intelectual; entre las personas con SPW hay una alta prevalencia de otras características más específicas en conducta y cognición como muestra la Tabla 1 (Butler, Lee y Whitman, 2006; Copet et al., 2010; Dimitropoulos, Ferranti y Lemler, 2013; Dimitropoulos, Ho y Feldman, 2013; Dimitropoulos y Schultz, 2007; Jauregi et al., 2007; Lo, Siemensma, Collin y Hokken-Koelega, 2013). De igual forma, el SPW afecta también al sistema familiar a nivel de padres, cuidadores principales y hermanos (Mazaheri et al., 2013). En resumen, el SPW tiene un gran impacto psicosocial que implica a la misma persona, su familia completa, instituciones educativas y su comunidad.

Según Wilson, Gracey, Evans y Bateman (2009) la rehabilitación neuropsicológica es un proceso que busca que las personas con déficits cognitivos, emocionales o conductuales logren su máximo potencial en diferentes dominios (psicológico, social, ocio, vocacional, habilidades de la vida diaria), todo basado en las interacciones cerebro-conducta y dirigido a alcanzar cambios funcionales. A pesar de que inicialmente solo se destacaba la rehabilitación cognitiva, hoy en día esta se considera solo una parte del proceso y a medida que sigue tomando fuerza el enfoque holístico se reconoce que la cognición, la emoción y la conducta están interrelacionadas (Wilson et al., 2009) y que, por lo tanto, el neuropsicólogo además de experto en cognición debe ser conocedor del funcionamiento emocional y de aspectos de personalidad, entre otros (Noggle, Dean y Barisa, 2013). Dentro de este contexto, la rehabilitación neuropsicológica incluye intervenciones multidisciplinarias que se pueden clasificar en cuatro grandes grupos: rehabilitación cognitiva, modificación de conducta, intervención con familias y readaptación vocacional o profesional (Muñoz-Marrón, 2009).

La rehabilitación neuropsicológica se basa en dos conceptos que revolucionaron la forma en que se concebía el cerebro y la organización de este: la plasticidad y la compensación estructural y funcional del cerebro. Cuando se vio que las personas con lesiones cerebrales en partes específicas eran capaces de recuperar las capacidades que se asociaban a esas áreas, se llegó a la consideración actual de que el cerebro trabaja de forma integrada y flexible, y que diferentes partes pueden encargarse de ciertas habilidades cuando la estructura primaria asociada es lesionada. Lo usual después de una lesión cerebral es que la recuperación siga un curso natural y espontáneo que es acelerado en los primeros momentos y se detiene meses después. Sin embargo, si esos procesos afectados se ven estimulados, la persona puede seguir recuperando funciones años después de haberse dado la lesión. Es en ese momento que toma un papel central la rehabilitación neuropsicológica como forma de estructurar estos esfuerzos de estimulación de forma personalizada para la persona afectada (Noggle et al., 2013).

En cuanto al diseño de estos esfuerzos terapéuticos, la rehabilitación neuropsicológica se basa en tres principios: (a) restauración: diseño de actividades sistemáticas 
para mejorar una capacidad cognitiva, (b) compensación: uso de las funciones cognitivas preservadas para apoyar las funciones perdidas mediante estrategias alternativas o ayudas externas, permitiéndole aprender o reaprender y (c) sustitución: mejorar el desempeño de la función alterada utilizando los sistemas conservados (Lubrini, Periañez y Ríos-Lago, 2009).

A medida que se conocen con mayor claridad las características y síntomas propios del SPW y sus bases neurológicas, se empieza a reconocer un terreno propicio para que se lleve a cabo un proceso de rehabilitación que se enfoque en este puente entre conducta/cognición y cerebro. De hecho, ya se empieza a aplicar en trastornos del neurodesarrollo, y no solo en déficits adquiridos, como es el caso de los Trastornos del Espectro Autista (Eack et al., 2013). Además, como enfoque útil para el tratamiento de personas con trastornos del neurodesarrollo está lo propuesto por Farran y Karmiloff-Smith (2011) en cuanto a que el perfil neuroconductual de estas personas no se debe limitar a una dicotomía de capacidades alteradas/preservadas, ya que aunque es útil en la práctica clínica, subestima el papel que la trayectoria personal de desarrollo ha tenido en esa persona y sus capacidades. Por otro lado, según estas autoras si solo se tienen en cuenta las capacidades etiquetadas como «alteradas», se deja de lado el gran potencial de las demás. Siguiendo este orden de ideas, en la Tabla 1 no solo se plasman las capacidades «alteradas», sino también las capacidades y dominios en los que se ha encontrado que las personas con SPW se destacan o que se conservan preservadas.

Dentro del SPW, el fenotipo neuroconductual tiene una serie de características propias, no obstante, gran parte de la literatura de intervenciones sobre el SPW se encuentra enfocada en los aspectos médicos (endocrinológicos, neurológicos, fisiológicos y nutricionales, entre otros) y muy poca en los conductuales y cognitivos. Adicionalmente, no se ha encontrado literatura que resuma y sintetice las intervenciones neuropsicológicas específicas que se han llevado a cabo para personas con SPW.

Esta revisión sistemática se propone debido a la relativa estabilidad del fenotipo neuroconductual, la trayectoria de desarrollo y necesidades únicas al SPW, y a la falta de documentación al respecto, lo que hace necesario responder a la pregunta de qué intervenciones sobre el fenotipo neuroconductual se están realizando actualmente en el campo de la rehabilitación neuropsicológica en personas con SPW. Lo anterior, con el fin de que los profesionales puedan planificar teniendo claro qué intervenciones están a la mano y así puedan responder más efectivamente a las necesidades de esta población con planes, programas y técnicas basadas en la evidencia.

\section{Método}

Esta revisión se hizo a través del análisis sistemático de los estudios que intervenían sobre variables cognitivas, conductuales y familiares en poblaciones de personas con SPW. En la Figura 1 se aprecian cada una de las fases y el proceso de la revisión partiendo de los lineamientos establecidos por Liberati et al. (2009). 


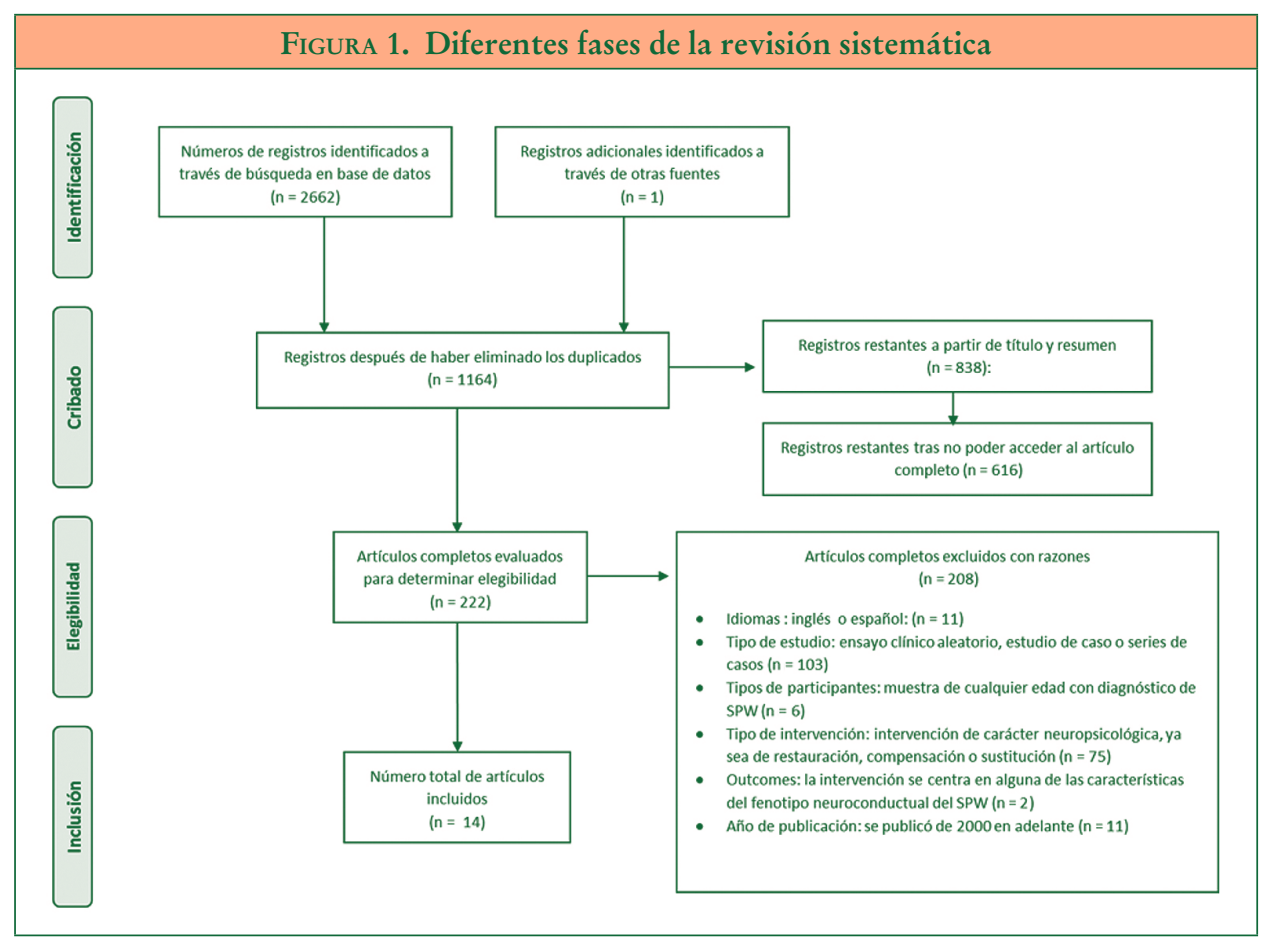

\subsection{Identificación de las publicaciones y procedimiento de búsqueda}

En primer lugar se aclara que en cada fase durante el procedimiento de búsqueda la única persona que intervino fue la autora del presente estudio.

El procedimiento de búsqueda se realizó en tres fases principales: 1) identificación de las publicaciones a utilizar a través de la búsqueda en diferentes bases de datos u otras fuentes; 2) cribado inicial de publicaciones a partir del título y el resumen, y la accesibilidad a la publicación, y 3 ) determinación de elegibilidad en la revisión sistemática a partir de los criterios de inclusión.

Para la fase de identificación de publicaciones se realizó una búsqueda en bases de datos donde no se impusieron límites de idioma ni origen. No obstante, se eligió limitar el rango de búsqueda desde 1980 hasta 2016 en las bases de datos que lo permitieran. Esta búsqueda fue aplicada en Science Direct, Scopus CIRRIE, PsycINFO, MEDLINE, Taylor y Francis, Wiley, Proquest, Pubpsych, EBMReviews and Cochrane, PubMed y ERIC. Los términos utilizados fueron: bebavio" (o behavior y behavioral), neuropsycholog* (o neuropsychology y neuropsychological), cognitive, neurocognitive, intervention, rehabilitation, management, treatment y Prader-Willi Syndrome. Los detalles de esta fase se encuentran disponibles en la Tabla 2. 
En la fase de cribado inicial se eliminaron los duplicados resultantes de la búsqueda inicial y luego, a partir de los títulos y resúmenes, se excluyeron los que no informaban de ningún tipo de intervención o tratamiento. Adicionalmente, como paso previo a la fase de elegibilidad se hizo otro cribado, excluyendo artículos a los que no se pudiera acceder al artículo completo a través de los recursos disponibles (bases de datos, préstamo interbibliotecario y comunicación con los autores).

\subsection{Fase de elegibilidad y criterios de inclusión}

Siguiendo las recomendaciones dispuestas en la Declaración PRISMA para revisiones sistemáticas y metaanálisis (Liberati et al., 2009), la elegibilidad de las publicaciones se evaluó basándose en características de los estudios y características de publicación. Específicamente, debían ser publicaciones que cumplieran los siguientes criterios: (a) idioma de la publicación: inglés o español; (b) tipo de estudio: ensayo clínico, estudio de cohorte, informe o series de casos; (c) tipos de participantes: muestra de cualquier edad que incluya explícitamente a personas con diagnóstico de SPW; (d) tipo de intervención: intervención de carácter neuropsicológico, ya sea de restauración, compensación o sustitución; (e) outcomes: la intervención se centra en alguna de las características del fenotipo neuroconductual del SPW (según la Tabla 1); (f) año de publicación: del año 2000 en adelante.

\subsection{Recolección de datos}

Los datos extraídos de los artículos incluidos en la revisión fueron: (a) modelo de rehabilitación (restauración, compensación, sustitución), (b) características de los participantes (número de participantes, edad media, sexo y presencia de discapacidad intelectual), (c) características fenotípicas del SPW que se está interviniendo (de la Tabla 1), (d) duración (diferentes fases temporales del estudio), (e) medidas (frecuencia, entre otras) y (d) resultados. Los datos a recolectar se establecieron a partir del objetivo de esta revisión, de modo que se puedan describir con detalle las intervenciones que se están realizando en rehabilitación neuropsicológica con personas con SPW.

\subsection{Calidad de los estudios}

De los estudios que finalmente fueron elegidos tras los cribados, todos son informes o series de casos correspondientes a los niveles más bajos de jerarquía en cuanto a niveles de evidencia ya que no había detalles del control adecuado de variables y de la metodología y en ninguno se utilizaron grupos de control a priori y en la mayoría se utilizó una muestra pequeña que podría no ser representativa de la población de personas con SPW. 


\begin{tabular}{|c|c|c|c|c|c|}
\hline \multicolumn{6}{|c|}{$\begin{array}{l}\text { Ejemplo de estrategia de búsqueda: SCIENCE DIRECT (filtrado 1980-presente) } \\
\text { [title-abs-key ("Prader-Willi Syndrome") AND (intervention OR rehabilitation OR treatment OR management) AND behavio"] }\end{array}$} \\
\hline & \multicolumn{5}{|c|}{ Bases de datos } \\
\hline & Science Direct & Scopus & CIRRIE & Psycinfo & Medline \\
\hline $\begin{array}{l}\text { Fecha de } \\
\text { búsqueda }\end{array}$ & 29/01/16-30/01/16 & 29/01/16-30/01/16 & $29 / 01 / 16-30 / 01 / 16$ & $29 / 01 / 16-30 / 01 / 16$ & $29 / 01 / 16-30 / 01 / 16$ \\
\hline $\begin{array}{r}\text { Términos } \\
\text { incluidos en } \\
\text { búsqueda }\end{array}$ & $\begin{array}{l}\text { PWS, } \\
\text { intervention, } \\
\text { rehabilitation, } \\
\text { treatment, } \\
\text { management, } \\
\text { behavior*, } \\
\text { cognitive, } \\
\text { neurocognitive, } \\
\text { neuropsycholog* }\end{array}$ & $\begin{array}{l}\text { PWS, } \\
\text { intervention, } \\
\text { rehabilitation, } \\
\text { treatment, } \\
\text { management, } \\
\text { behavior*, } \\
\text { cognitive, } \\
\text { neurocognitive, } \\
\text { neuropsycholog* }\end{array}$ & $\begin{array}{l}\text { PWS, } \\
\text { intervention, } \\
\text { rehabilitation, } \\
\text { treatment, } \\
\text { management, } \\
\text { behavior, } \\
\text { behavioral, } \\
\text { cognitive, } \\
\text { neurocognitive, } \\
\text { neuropsychology, } \\
\text { neuropsychological }\end{array}$ & $\begin{array}{l}\text { PWS, } \\
\text { intervention, } \\
\text { rehabilitation, } \\
\text { treatment, } \\
\text { management, } \\
\text { behavior, } \\
\text { cognitive, } \\
\text { neurocognitive, } \\
\text { neuropsychology, } \\
\text { neuropsychological }\end{array}$ & $\begin{array}{l}\text { PWS, } \\
\text { intervention, } \\
\text { rehabilitation, } \\
\text { treatment, } \\
\text { management, } \\
\text { behavior, } \\
\text { cognitive, } \\
\text { neurocognitive, } \\
\text { neuropsychology, } \\
\text { neuropsychological }\end{array}$ \\
\hline $\begin{array}{l}\text { Limitaciones de } \\
\text { la búsqueda }\end{array}$ & $\begin{array}{l}\text { Título, resumen, } \\
\text { palabras clave } \\
\text { 1980-presente }\end{array}$ & $\begin{array}{l}\text { Título, resumen, } \\
\text { palabras clave } \\
1980-2016\end{array}$ & $1990-2016$ & $1980-2016$ & $1980-2016$ \\
\hline $\begin{array}{r}\text { Publicaciones } \\
\text { encontradas }\end{array}$ & 480 & 527 & 17 & 197 & 178 \\
\hline
\end{tabular}

Nota. PWS = Prader-Willi Syndrome

\section{Resultados}

\subsection{Análisis de la información}

Se dividieron los 14 artículos en estudios que intervienen sobre la hiperfagia o conductas relacionadas y estudios que intervienen sobre otras características cognitivas, conductuales o familiares de la Tabla 1.

\subsection{Hallazgos generales}

La Tabla 3 resume los resultados encontrados. 


\begin{tabular}{|c|c|c|c|c|c|}
\hline Taylor y Francis & Wiley & Proquest & $\begin{array}{l}\text { EBM REVIEWS+ } \\
\text { Cochrane }\end{array}$ & Pubmed & ERIC \\
\hline 29/01/16-30/01/16 & $29 / 01 / 16-30 / 01 / 16$ & 29/01/16-30/01/16 & $29 / 01 / 16-30 / 01 / 16$ & $29 / 01 / 16-30 / 01 / 16$ & $29 / 01 / 16-30 / 01 / 16$ \\
\hline $\begin{array}{l}\text { PWS, } \\
\text { intervention, } \\
\text { rehabilitation, } \\
\text { treatment, } \\
\text { management, } \\
\text { behavior, } \\
\text { cognitive, } \\
\text { neurocognitive, } \\
\text { neuropsychology, } \\
\text { neuropsychological }\end{array}$ & $\begin{array}{l}\text { PWS, } \\
\text { intervention, } \\
\text { rehabilitation, } \\
\text { treatment, } \\
\text { management, } \\
\text { behavior, } \\
\text { cognitive, } \\
\text { neurocognitive, } \\
\text { neuropsychology, } \\
\text { neuropsychological }\end{array}$ & $\begin{array}{l}\text { PWS, } \\
\text { intervention, } \\
\text { rehabilitation, } \\
\text { treatment, } \\
\text { management, } \\
\text { behavior, } \\
\text { cognitive, } \\
\text { neurocognitive, } \\
\text { neuropsychology, } \\
\text { neuropsychological }\end{array}$ & $\begin{array}{l}\text { PWS, } \\
\text { intervention, } \\
\text { rehabilitation, } \\
\text { treatment, } \\
\text { management, } \\
\text { behavior, } \\
\text { cognitive, } \\
\text { neurocognitive, } \\
\text { neuropsychology, } \\
\text { neuropsychological }\end{array}$ & $\begin{array}{l}\text { PWS, } \\
\text { intervention, } \\
\text { rehabilitation, } \\
\text { treatment, } \\
\text { management, } \\
\text { behavior, } \\
\text { cognitive, } \\
\text { neurocognitive, } \\
\text { neuropsychology, } \\
\text { neuropsychological }\end{array}$ & $\begin{array}{l}\text { PWS, } \\
\text { intervention, } \\
\text { rehabilitation, } \\
\text { treatment, } \\
\text { management, } \\
\text { behavior, } \\
\text { cognitive, } \\
\text { neurocognitive, } \\
\text { neuropsychology, } \\
\text { neuropsychological }\end{array}$ \\
\hline $1980-2016$ & $\begin{array}{l}\text { Resumen, } \\
\text { 1980-2016 }\end{array}$ & $\begin{array}{l}\text { Resumen, } \\
\text { 1980-2016 }\end{array}$ & & $1980-2016$ & 1997-2016 \\
\hline 601 & 59 & 206 & 13 & 297 & 38 \\
\hline
\end{tabular}




\begin{tabular}{|c|c|c|c|c|c|c|c|}
\hline \multirow{8}{*}{ 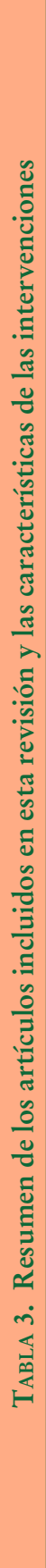 } & 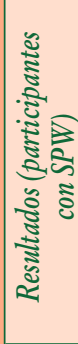 & 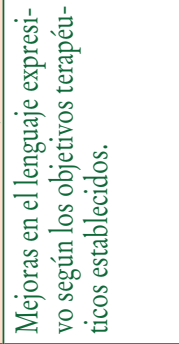 & 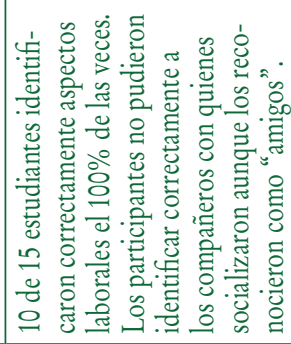 & 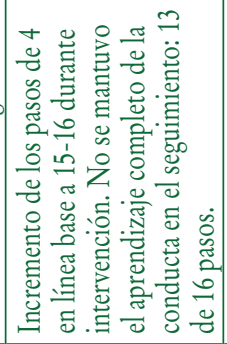 & 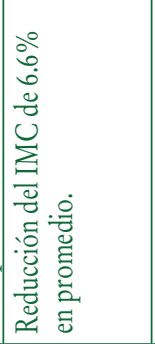 & 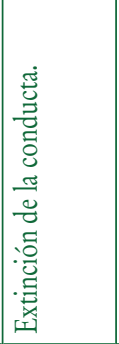 & 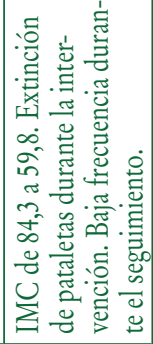 \\
\hline & $\frac{3}{3}$ & 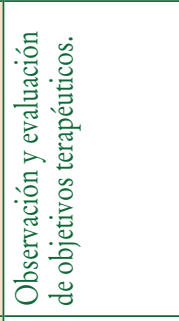 & 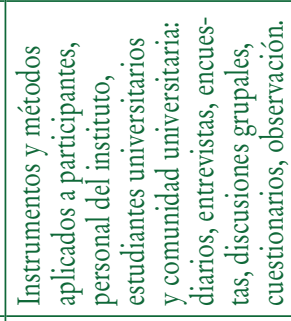 & 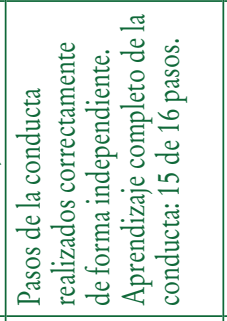 & $\begin{array}{l}\sum_{i}^{0} \\
0 \\
0 \\
0 \\
0\end{array}$ & 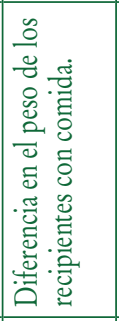 & 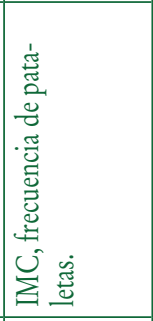 \\
\hline & 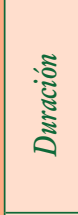 & 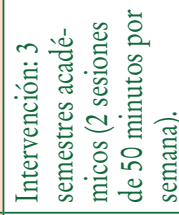 & 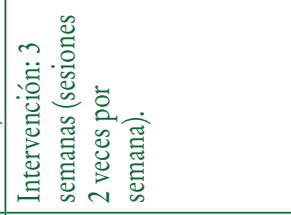 & 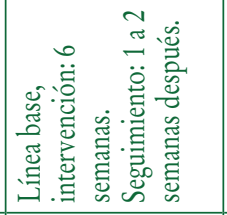 & 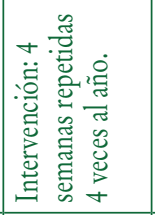 & 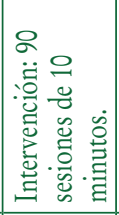 & 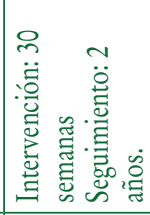 \\
\hline & 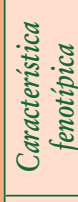 & 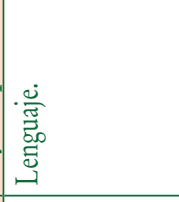 & 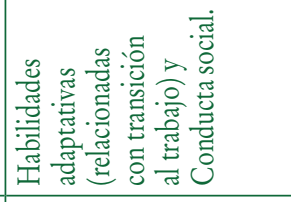 & 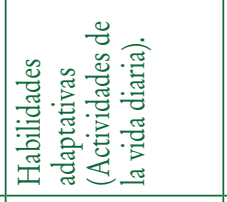 & 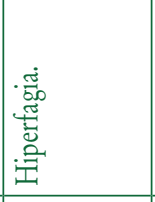 & 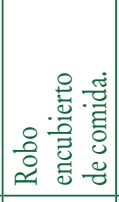 & 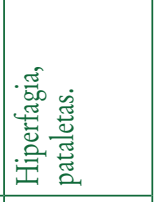 \\
\hline & 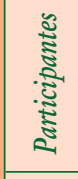 & 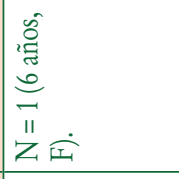 & 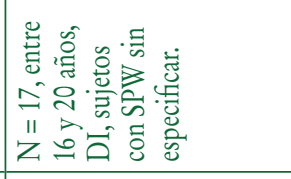 & 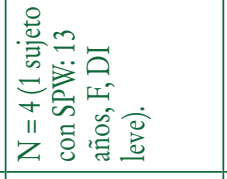 & 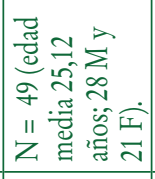 & 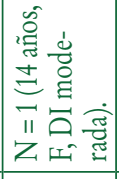 & 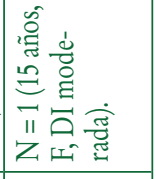 \\
\hline & 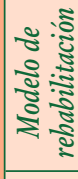 & 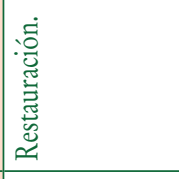 & 离: & 离: & 竞: & 竞: & 悹:施 \\
\hline & 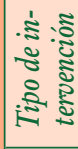 & 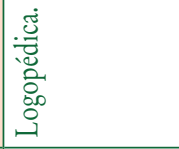 & 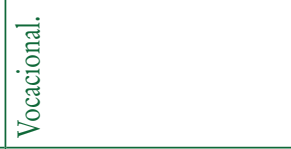 & 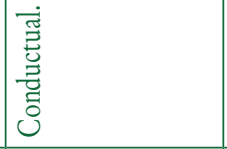 & 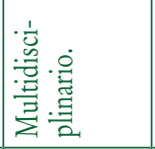 & 珳 & 胥 \\
\hline & 胥 & 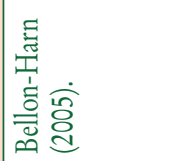 & 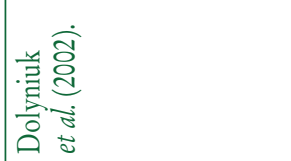 & 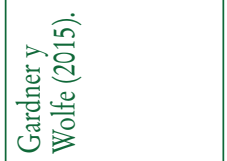 & 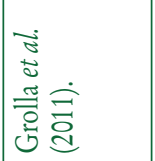 & 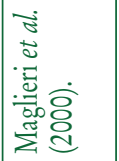 & 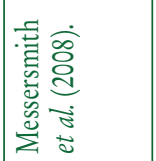 \\
\hline
\end{tabular}

(c) Ediciones Universidad de Salamanca / CC BY-NC-ND

Siglo Cero, vol. 48 (1), n. ${ }^{\circ}$ 261, 2017, enero-marzo, pp. 73-93 


\begin{tabular}{|c|c|c|c|c|c|c|c|c|c|c|}
\hline \multirow{8}{*}{ 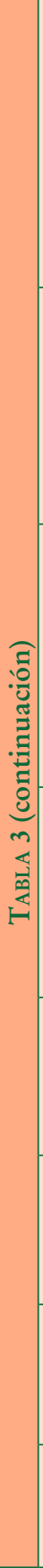 } & 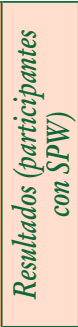 & 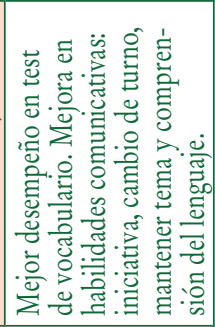 & 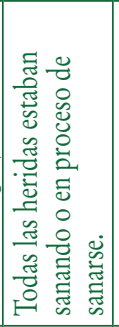 & 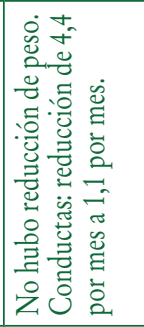 & 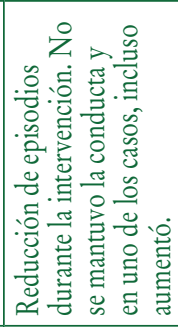 & 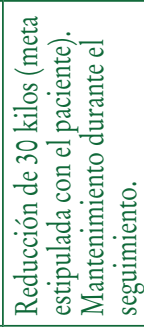 & 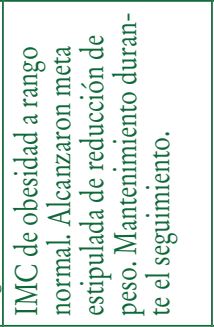 & 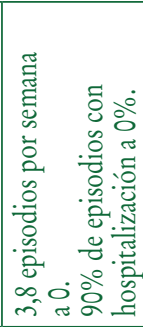 & 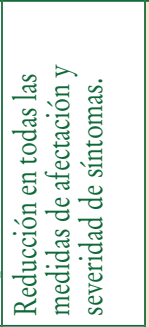 & \\
\hline & 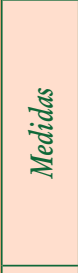 & 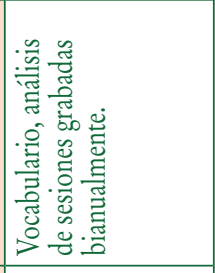 & 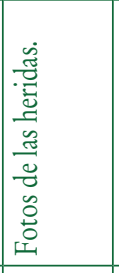 & 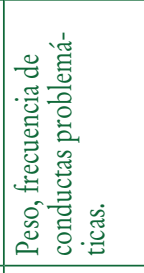 & 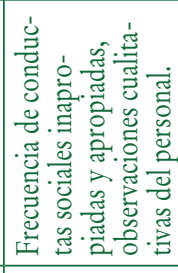 & \begin{tabular}{|l}
0 \\
0 \\
0 \\
0 \\
0 \\
0 \\
0 \\
0
\end{tabular} & $\begin{array}{l}0 \\
\vdots \\
\vdots \\
0 \\
0 \\
0 \\
\end{array}$ & 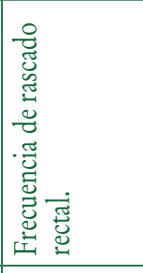 & 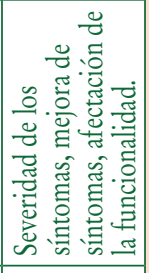 & \\
\hline & 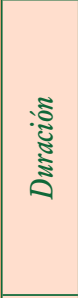 & 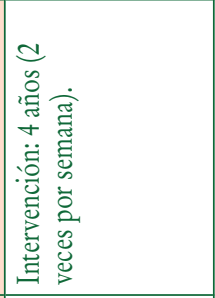 & 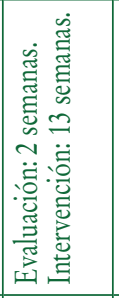 & 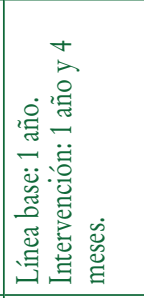 & 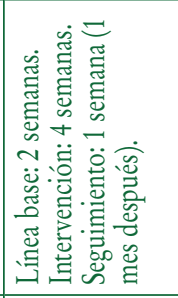 & 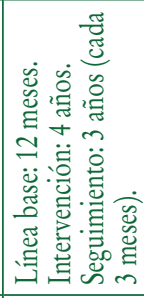 & 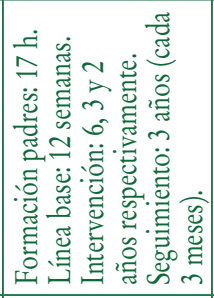 & 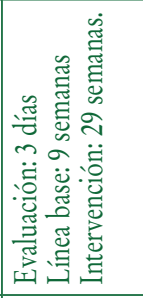 & 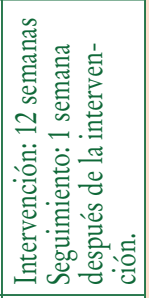 & 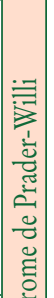 \\
\hline & 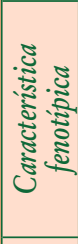 & 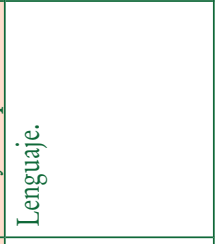 & 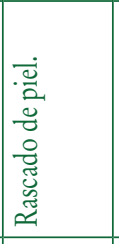 & 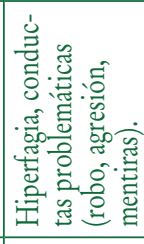 & 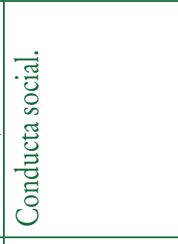 & 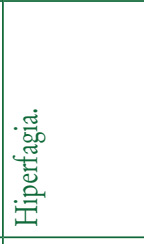 & 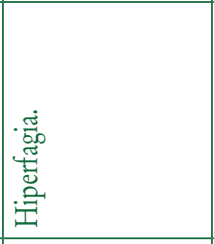 & 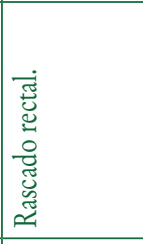 & 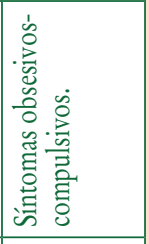 & 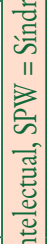 \\
\hline & 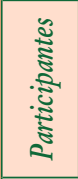 & 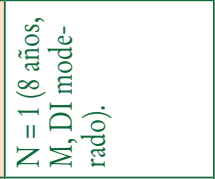 & 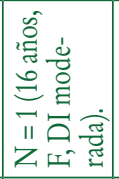 & 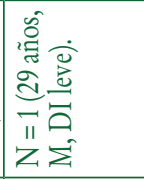 & 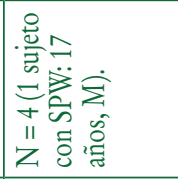 & 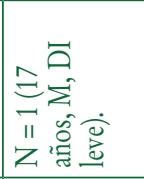 & 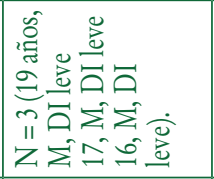 & 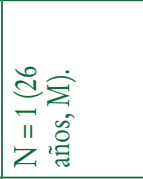 & 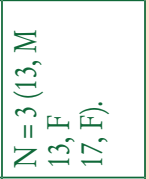 & 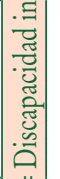 \\
\hline & 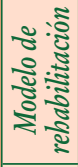 & 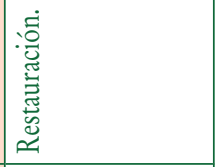 & 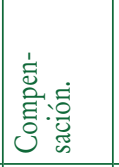 & 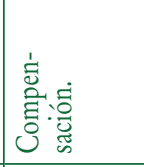 & 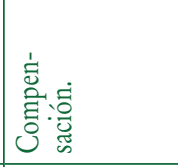 & 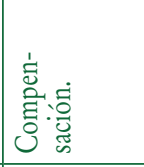 & 空: & 离 & 空: & 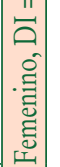 \\
\hline & 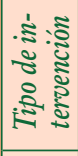 & 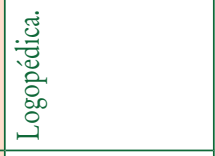 & 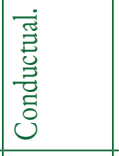 & 搃密 & 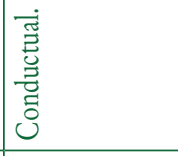 & 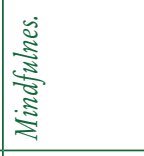 & 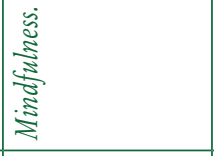 & 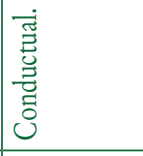 & 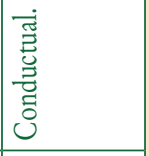 & 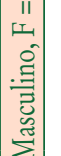 \\
\hline & 荵 & 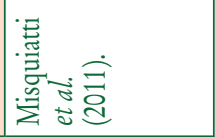 & 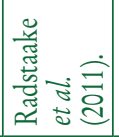 & 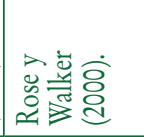 & 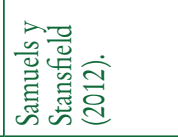 & 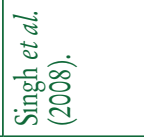 & 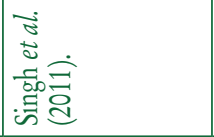 & 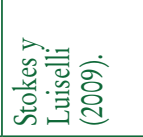 & 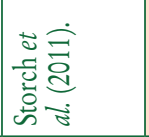 & $\begin{array}{l}\sum^{11} \\
5 \\
z \\
z\end{array}$ \\
\hline
\end{tabular}

() Ediciones Universidad de Salamanca / CC BY-NC-ND

Siglo Cero, vol. 48 (1), n. ${ }^{\circ}$ 261, 2017, enero-marzo, pp. 73-93 


\section{Participantes}

En total, en los 14 estudios hubo 88 participantes. Las muestras de estos estudios eran pequeñas (6,2 de media, rango: 1 a 49) y la mayoría se compuso de niños, jóvenes y adultos jóvenes entre los 6 y los 29 años. Con excepción de tres estudios (Dolyniuk et al., 2002; Gardner y Wolfe, 2015; Samuels y Stansfield, 2012), todos los demás intervenían exclusivamente a personas con SPW.

En cuanto al nivel intelectual, todos estuvieron en algún punto entre inteligencia límite o discapacidad intelectual moderada. Varios estudios no dieron información detallada sobre el funcionamiento intelectual de sus participantes (Bellon-Harn, 2005; Grolla et al., 2011; Samuels y Stansfield, 2012; Storch et al., 2011; Stokes y Luiselli, 2009). Por otro lado, el estudio de Dolyniuk et al. (2002) no dio ningún tipo de información detallada de su muestra.

\section{Intervenciones}

Las características fenotípicas intervenidas fueron lenguaje (Bellon-Harn, 2005; Misquiatti, Cristovão y Brito, 2011), habilidades adaptativas (Dolyniuk et al., 2002; Gardner y Wolfe, 2015), hiperfagia (Grolla et al., 2011; Messersmith, Slifer, Pulbrook-Vetter y Bellipanni, 2008; Rose y Walker, 2000; Singh et al., 2008; Singh et al., 2011), robo de comida (Maglieri, DeLeon, Rodriguez-Catter y Sevin, 2000), conducta social (Samuels y Stansfield, 2012), pataletas (Messersmith et al., 2008; Rose y Walker, 2000), rascado de piel (Radstaake et al., 2011), rascado rectal (Stokes y Luiselli, 2009) y síntomas obsesivos-compulsivos (Storch et al., 2011).

En cuanto a las fases, además de la intervención, seis estudios explicitaron una fase de línea base (Rose y Walker, 2000; Stokes y Luiselli, 2009), un estudio explicitó una fase de seguimiento (Messersmith et al., 2008), cuatro estudios explicitaron tanto fase de línea base como de seguimiento (Gardner y Wolfe, 2015; Samuels y Stansfield, 2012; Singh et al., 2008; Singh et al., 2011) y siete estudios no explicitaron ninguna de las dos (Bellon-Harn, 2005; Dolyniuk et al., 2002; Grolla et al., 2011; Maglieri et al., 2000; Misquiatti et al., 2011; Radstaake et al., 2011; Storch et al., 2011).

El $43 \%$ de los estudios utilizaron intervenciones de corte puramente conductual y el resto fueron logopédicas, vocacionales, mindfulness y cognitivo-conductual. La mayoría de las intervenciones se realizaron en algún centro, hospital o institución clínica, con excepción de cinco estudios que se realizaron en casa de los sujetos, parcialmente en pisos tutelados o en la escuela (Dolyniuk et al., 2002; Gardner y Wolfe, 2015; Radstaake et al., 2011; Singh et al., 2008; Singh et al., 2011).

\section{Modelo de rehabilitación}

La mayoría de los estudios encontrados corresponden al modelo de compensación, ya que no se pretende mejorar funciones en sí, sino que, a través de adaptaciones 
externas y entrenamientos, buscan mejorar la funcionalidad de la persona (Lubrini et al., 2009). Por un lado, los enfoques de tipo conductual implican un entrenamiento en habilidades específicas y una modificación del entorno y acomodación o ajuste de las tareas en el entorno. Por otro lado, en el caso de los estudios que utilizaron el mindfulness y el enfoque cognitivo-conductual, hay un especial énfasis en el entrenamiento en estrategias metacognitivas que en este caso se usan para lograr un mayor control personal. De igual forma, en el programa vocacional de Dolyniuk et al. (2002) se buscaba que se dieran aprendizajes de habilidades adaptativas enfocadas al mundo laboral.

Los dos estudios que usaron el modelo de restitución (Bellon-Harn, 2005; Misquiatti et al., 2011) fueron intervenciones logopédicas que buscaban que los sujetos desarrollaran o recuperaran diferentes aspectos de la función del lenguaje.

\subsection{Proceso de intervención}

Dentro de este apartado se tienen en cuenta las características del proceso de intervención.

\section{Estudios que intervienen sobre la hiperfagia}

En la revisión bibliográfica se hizo evidente que ningún estudio apunta explícitamente al tratamiento de la hiperfagia desde un enfoque de rehabilitación. En los tres estudios se habla de «control de peso» en el que además de un régimen alimenticio o de ejercicio, se aplican técnicas que buscan que el paciente respete dicho régimen.

\subsection{Procedimientos de intervención y resultados}

Grolla et al. (2011) reflejan en su estudio los resultados de más de seis años de ciclos trimestrales de rehabilitación con el fin de controlar el peso y el índice de masa corporal (IMC). Los ciclos tenían una duración de 4 semanas y en la muestra había pacientes que habían tenido diferentes niveles de participación (más de tres ciclos consecutivos, no consecutivos y menos de tres). La intervención se hizo en grupos de menos de 10 sujetos, llevándose un régimen alimenticio de $1.500 \mathrm{kc}, 6 \frac{1}{2} 2$ horas de ejercicios diarios y 2 horas diarias de terapia musical y psicomotora. Los resultados muestran una reducción del IMC del $6,6 \%$ en promedio.

Singh et al. (2008) y Singh et al. (2011) utilizaron la técnica de mindfulness para reducir y mantener el peso. En ambos estudios se midió el peso y el IMC, y se hizo la intervención en casa, con las madres como terapeutas y con apoyo a través de Internet. En el caso de Singh et al. (2008) se realizó en 8 años y se dividió en tres fases: (1) ejercicio por 30 minutos diarios; (2) ejercicio por 30 minutos y seguimiento de un programa alimenticio; y (3) ejercicio, programa alimenticio y además ejercicios 
de mindfulness que consistían en: mejora de hábitos alimenticios, visualización y meditación. En Singh et al. (2011) se utilizaron las mismas técnicas pero, en lugar de dividirse en fases, se integró todo en una fase de intervención, seguida por un seguimiento. En ambos los resultados indicaron que el tratamiento fue exitoso, logrando disminuir significativamente el peso, manteniéndose estos resultados durante la fase de seguimiento.

\section{Estudios con intervención sobre hiperfagia y otra característica fenotípica}

Hubo dos estudios donde se intervino sobre la hiperfagia y al mismo tiempo otra característica fenotípica del SPW.

\subsection{Procedimientos de intervención y resultados}

Messersmith et al. (2008) trabajaron multidisciplinariamente para reducir el peso y las pataletas. La intervención tuvo una duración de 2 años y 5 meses divididos en intervención y seguimiento en los cuales se siguió un régimen alimenticio, terapia física y ocupacional, establecimiento de rutinas, economía de fichas y entrenamiento conductual a padres; utilizando técnicas como extinción social, prevención de escape, manejo de antecedentes y reforzamiento diferencial de conductas alternativas. Los resultados demuestran extinción de las pataletas y una reducción del peso corporal con mantenimiento de los resultados durante el seguimiento.

Rose y Walker (2000) apuntaron a reducir el peso y conductas inadecuadas (daño a la propiedad, robo, abuso verbal y físico) usando la técnica de entrevista emocional, reforzamiento y programación positiva. Tuvo una duración de 29 meses divididos en línea base e intervención. Se entrenó al equipo de tutelaje sobre el manejo de las conductas con el uso de empatía, evasión de confrontaciones, apoyo a la autoeficacia, entre otras. Los resultados muestran que la intervención no tuvo impacto en el peso, sin embargo, las conductas problemáticas disminuyeron considerablemente.

\section{Estudios con intervención sobre otras características fenotípicas}

Hubo nueve estudios donde se intervino explícitamente alguna característica fenotípica del SPW (aparte de la hiperfagia), tomándola como medida dependiente de la intervención.

\subsection{Procedimientos de intervención y resultados}

Bellon-Harn (2005) realizó una intervención específica del lenguaje en una persona con dificultades severas en el lenguaje expresivo. Durante la intervención se 
establecieron objetivos en conjunto con la familia y se trabajaba con la logopeda y en casa. En las sesiones se trabajó con ejercicios explícitos de estimulación multisensorial, terapia de producción oral, herramientas para identificar emisiones nasales de aire, libros de cuentos, entre otros. El resultado de la intervención fue una mejora consistente pero lenta del lenguaje expresivo de la paciente, sin embargo, hacia el final de la intervención se determina que es necesario empezar a hacer uso de un enfoque compensatorio con tecnología de apoyo para la comunicación.

Dolyniuk et al. (2002) crearon un programa vocacional para estudiantes de instituto con discapacidad intelectual que tenían que enfrentarse pronto a la vida laboral. En este programa se implicó no solo a los participantes, sino a personas de la comunidad universitaria (estudiantes universitarios, profesores) en la que los participantes debían aprender información y habilidades útiles para una futura inclusión laboral. El programa consistía en que los participantes rotaban por diferentes zonas de la universidad vinculadas a diferentes oficios en sesiones donde podían aprender qué se hacía y cómo. El programa tenía como objetivo indirecto reforzar la conducta social de los participantes hacia sus pares y para esto se escogió a estudiantes universitarios como mentores y apoyos naturales para los participantes. Los resultados del programa muestran que hubo aprendizaje de información clave en el aspecto vocacional, pero en el aspecto social no pudieron demostrar ninguna mejora.

Gardner y Wolfe (2015) utilizaron vídeos para enseñar una actividad de la vida diaria a estudiantes en el ambiente natural de una cocina dentro de la escuela. La conducta «lavar platos» se dividió en 16 pasos. Se realizó una fase de línea base y a continuación la intervención en la que los participantes debían observar un vídeo de un paso y llevarlo a cabo con posibilidad de ayudas adicionales (modelo o ayuda física). Se consideraba que la conducta estaba aprendida si se realizaban 15 pasos sin ayudas adicionales durante cuatro sesiones. Todos los sujetos consiguieron aprender la conducta objetivo y específicamente en la persona con SPW, aunque se dio este aprendizaje, no se mantuvo en la fase de seguimiento, llegando solo a 13 pasos aprendidos de 16 .

Maglieri et al. (2000) aplicaron técnicas conductuales para disminuir el robo encubierto de comida a través del control de estímulo y castigo, midiéndolo al comparar los pesos de los contenedores de comida en sesiones de 10 minutos de duración. En las primeras sesiones, se castigó de forma verbal la conducta de robo y en las sesiones de control de estímulo se utilizaron etiquetas para determinar qué comida estaba prohibido comer y cuál no, y en caso de que comiera algo prohibido se daba una reprimenda verbal. Hubo una fase de generalización en la cocina, utilizando las despensas y el refrigerador. La conducta se extinguió y se mantuvieron los resultados durante el seguimiento.

Misquiatti et al. (2011) realizaron una intervención logopédica en un paciente con dificultades en el lenguaje expresivo y comprensivo. La intervención se realizó desde la perspectiva pragmática, dando relevancia a la relación con la logopeda y con actividades lúdicas. Los resultados de la intervención demostraron mejoras en la intención comunicativa y en la capacidad de mantener una conversación, sin embargo, se mantuvieron las dificultades en el lenguaje comprensivo y dificultades perceptuales que 
no permitieron llegar al punto de comprender lenguaje más complejo para desarrollar capacidades conversacionales y narrativas acordes a su edad.

Radstaake et al. (2011) utilizaron el enfoque conductual para reducir el rascado de piel a través de reforzamiento diferencial de conductas alternativas y reforzamiento diferencial de conductas incompatibles. Como medida se tomaron fotos de la progresión de las heridas y se les pidió a unos jueces que las ordenaran para determinar el efecto de la intervención. Este estudio constó de dos fases: evaluación y tratamiento, en las cuales se realizó una evaluación funcional y se propuso la aplicación de loción y juego con un peluche/libro como conductas a reforzar. Se utilizó en mayor medida el refuerzo social y se hicieron sesiones privadas con la paciente como seguimiento. El proceso fue exitoso, logrando disminuir la conducta y las heridas, manteniéndose hasta un mes después.

Samuels y Stansfield (2012) aplicaron la técnica de historias sociales a sujetos con dificultades a nivel social con el fin de disminuir conductas identificadas como problemáticas de parte del personal de la residencia donde vivían. Las historias sociales fueron diseñadas enfocándose a dichas conductas y se leían previamente al momento en que probablemente se iban a poder aplicar. En la persona con SPW, aunque hubo una reducción de las conductas objetivo durante la intervención, posteriormente no solo no se mantuvo, sino que superó la frecuencia de ocurrencia encontrada en la línea base.

Stokes y Luiselli (2009) utilizaron el análisis conductual aplicado para disminuir el rascado rectal. La intervención duró 38 semanas, divididas en línea base e intervención. Las técnicas fueron la limitación del tiempo en el baño, entrenamiento en comunicación funcional y el reforzamiento positivo diferencial. La conducta disminuyó de 3,8 incidentes por semana (90\% requiriendo hospitalización) a cero durante la intervención y en ningún episodio se requirió hospitalización.

Storch et al. (2011) utilizaron la terapia conductual para disminuir los síntomas ritualistas, midiendo con una serie de escalas de diagnóstico psiquiátrico la severidad de los síntomas, mejora de los síntomas e impacto de los síntomas en la funcionalidad. La intervención se realizó en 12 sesiones semanales enfocadas en la familia en las que se dio un entrenamiento en manejo de conducta a padres, que incluían técnicas como economía de fichas, reforzamiento diferencial y tiempos fuera. Los resultados mostraron una disminución en los síntomas, su severidad y su impacto en la funcionalidad.

\section{Discusión y conclusiones}

Este trabajo tenía como objetivo realizar una revisión sistemática de las intervenciones neuropsicológicas en personas con SPW, lo cual ha sido posible de abordar a partir de la búsqueda sistemática y los consecuentes resultados obtenidos. Como observación, esta revisión estuvo limitada por el pequeño número de estudios de intervenciones de SPW en el campo de la neuropsicología y por el hecho de que los existentes en su gran mayoría son estudios de casos con menos de cinco participantes y con una metodología poco rigurosa. 
Se destaca que las intervenciones son congruentes con lo que se sabe del desarrollo del fenotipo y sus fases porque se concentran en las edades en que se espera que la fase hiperfágica empiece o presente la mayoría de dificultades conductuales, con pocos estudios con sujetos sobre los treinta años, lo cual se puede explicar gracias a los hallazgos en la literatura que demuestran contradicciones en cuanto a las conductas problemáticas de los 30 años en adelante (Pignatti et al., 2013). De hecho, en el único estudio que se incluyó a una persona con SPW menor de ocho años, esta no presentaba ninguna alteración conductual o emocional (Bellon-Harn, 2005).

Casi todas las intervenciones tienen un enfoque compensador que apunta a mejorar la funcionalidad a través de la modificación conductual, lo cual va de la mano con los estudios que han hallado que las conductas más problemáticas como las pataletas o los comportamientos ritualistas se deben manejar con técnicas conductuales (Goldstone et al., 2008). Sin embargo, no se hallaron intervenciones que se centraran en las características neurocognitivas o familiares encontradas en el SPW. A pesar de la abundante literatura sobre las características fenotípicas neurocognitivas, solo se hacen descripciones de estas sin ninguna propuesta rehabilitadora. En general, las intervenciones se siguen manteniendo en el modelo deficitario pues solo dos estudios contemplaron las fortalezas cognitivas o conductuales presentes en el SPW (Whittington, 2004). Aunque se encuentra poca diversidad en cuanto a los objetivos de las intervenciones (mayor interés por hiperfagia y conductas asociadas), sí se han tenido en cuenta algunas habilidades relacionadas con el trabajo que los padres han declarado como de alta prioridad (Mazaheri et al., 2013).

En cuanto a las intervenciones, se observa que solo Maglieri et al. (2000) utilizan el castigo, yendo en la línea de la gran mayoría de los estudios de los últimos años que se concentran en el reforzamiento diferencial de la conducta usando reforzadores sociales y promoviendo la participación del sujeto en la intervención, haciéndole protagonista activo de su proceso, incluso permitiéndole determinar sus objetivos de peso y el ritmo de consecución de las metas como Singh et al. (2008) y Singh et al. (2011).

Por otro lado, entre los estudios, se observa que se implica poco a la familia en las intervenciones ya que solo tres estudios dieron importancia al apoyo familiar, lo cual ha demostrado ser un factor crucial, ya que la gran mayoría de las familias quiere una guía sobre el manejo de conflictos con las personas con SPW en su vida diaria (Wigren y Hansen, 2003).

Uno de los resultados que más llaman la atención son los de Singh et al. (2008) y Singh et al. (2011), cuyos estudios son los que obtienen los mejores resultados en cuanto al manejo del peso y mantenimiento de los resultados, y porque implican que con entrenamiento algunas personas con SPW pueden lograr tener cierto nivel de autocontrol sobre la ingesta alimenticia. Esto además va de la mano con la tendencia actual de encontrar un equilibrio en las intervenciones para promover la autorrealización en personas con SPW (Van Hooren, Widdershoven, Candel, Van den Borne y Curfs, 2006).

La mayoría de las intervenciones implicaron sacar al sujeto de su ambiente natural y enviarlo a instalaciones especializadas durante la intervención, aunque algunas realizaron seguimiento y programaron una implementación en el hogar, lo que puede 
explicar las dificultades para generalizar y mantener los resultados fuera del ambiente terapéutico y podría también explicar el mantenimiento en el caso de Singh et al. (2008) y Singh et al. (2011).

Otro punto a resaltar es que en contados casos se hace referencia al tipo de SPW, yendo en contravía a la tendencia actual de diferenciar las anomalías cromosómicas que han dado origen al síndrome, especialmente entre el tipo de deleción (Tipo I y II) o disomía uniparental materna, ya que parece que el fenotipo característico cambia o tiene tendencias a marcar más unas características que otras dependiendo del subtipo genético exhibido (Whittington, 2004).

En conclusión, se encuentra que, en cuanto a los aspectos procedimentales en general, los estudios demuestran poca homogeneidad, no coincidiendo en temporalidad, localización, ni profesionales que intervienen; así como en aspectos más específicos como tipo de reforzadores, tipo de estrategias conductuales, entre otros. Además, se hace difícil generalizar los resultados por falta de controles, control de variables, detalles de las intervenciones y métodos, entre otros. Esto hace que se dificulte el poder evaluar todas las intervenciones al mismo nivel pues las discrepancias son muchas y dificulta más aún la aplicación informada de los terapeutas en su práctica clínica.

No obstante, lo que se puede aseverar es que las intervenciones de tipo conductual tienen un valor terapéutico importante y que son especialmente efectivas cuando se da preferencia al reforzamiento diferencial. También se ve la importancia de implicar al sujeto y su familia dentro del proceso de rehabilitación, y de procurar que las intervenciones se realicen en el ambiente natural del sujeto.

Los resultados de esta revisión ponen en evidencia que es necesario ampliar las perspectivas de intervención en cuanto al SPW, ya que el modelo deficitario utilizado por la mayoría de los estudios de intervención de esta revisión lleva a proponer una intervención de tipo compensatorio, dejando de lado en gran medida las fortalezas que presentan estas personas. Asimismo, es necesario que se documenten más casos clínicos de diferentes ámbitos terapéuticos que utilicen propuestas sustitutivas y restitutivas, así como estudios con metodologías más rigurosas que puedan proporcionar realmente prácticas basadas en evidencias y hallazgos relevantes.

Por último, son necesarias más investigaciones en el campo de la rehabilitación en casos de fenotipos neuroconductuales tan marcados como los encontrados en el SPW y sus opciones de intervención a nivel conductual, cognitivo y familiar, de modo que se incluya un enfoque de fortalezas y debilidades que realmente pueda satisfacer las necesidades de rehabilitación tanto para familias como para las mismas personas con SPW.

\section{Referencias bibliográficas}

Bellon-Harn, M. (2005). Clinical management of a child with Prader-Willi Syndrome from maternal uniparental disomy (UPD) genetic inheritance. Journal of Communication Disorders, 38, 459-472. doi:10.1016/j.jcomdis.2005.04.001.

Butler, M., Lee, P. y Whitman, B. (Eds.) (2006). Management of Prader-Willi Syndrome. New York: Springer. 
Copet, P., Jauregi, J., Laurier, V., Ehlinger, V., Arnaud, C., Cobo, A., Molinas, C., TauBER, M. y Thuilleaux, D. (2010). Cognitive profile in a large french cohort of adults with Prader-Willi syndrome: differences between genotypes. Journal of Intellectual Disability Research, 54 (3), 204-215. doi:10.1111/j.1365-2788.2010.01251.x.

Dimitropoulos, A., Ferranti, A. y Lemler, M. (2013). Expressive and receptive language in Prader-Willi syndrome: Report on genetic subtype differences. Journal of Communication Disorders, 46 (2), 193-201. doi:10.1016/j.jcomdis.2012.12.001.

Dimitropoulos, A., Ho, A. y Feldman, B. (2013). Social Responsiveness and Competence in Prader-Willi Syndrome: Direct Comparison to Autism Spectrum Disorder. Journal of Autism y Developmental Disorders, 43 (1), 103-113. doi:10.1007/s10803-012-1547-3.

Dimitropoulos, A. y Schultz, R. T. (2007). Autistic-like symptomatology in Prader-Willi syndrome: a review of recent findings. Current Psychiatry Reports, 9 (2), 159-164.

Dolyniuk, C., Kamens, M., Corman, H., Dinardo, P., Totaro, R. y Rockoff, J. (2002). Students with developmental disabilities go to college: Description of a collaborative transition project on a regular college campus. Focus on Autism and Other Developmental Disabilities, 17 (4).

Eack, S. M., Bahorik, A. L., Hogarty, S. S., Greenwald, D. P., Litschge, M. Y., Mazefsky, C. A. y Minshew, N. J. (2013). Is Cognitive Rehabilitation Needed in Verbal Adults with Autism? Insights From Initial Enrollment in a Trial of Cognitive Enhancement Therapy. Journal of Autism and Developmental Disorders, 43 (9), 2233-2237. http://doi.org/10.1007/ s10803-013-1774-2.

Farran, E. y Karmiloff-Smith, A. (Eds.) (2011). Neurodevelopmental Disorders Across the Lifespan: A neuroconstructivist approach. Oxford: Oxford University Press. Extraído desde:http:// www.oxfordscholarship.com/view/10.1093/acprof:oso/9780199594818.001.0001/acprof-9780199594818.

Flint, J. y Yule, W. (1994). Behavioural phenotypes. En M. R. RutTer, E. TAylor y L. Hersov (Eds.), Child and Adolescent Psychiatry (pp. 666-687). Oxford: Blackwell Scientific.

Gardner, J. y Wolfe, P. (2015). Teaching students with developmental disabilities daily living skills using point-of-view modeling plus video prompting with error correction. $F_{O}-$ cus on Autism and Other Developmental Disabilities, 30 (4), 195-207. doi:10.1177/10883 57614547810.

Goldstone, A. P., Holland, A. J., Hauffa, B. P., Hokken-Koelega, A. C., Tauber, M. y Speakers contributors at the Second Expert Meeting of the Comprehensive Care of Patients with PWS (2008). Recommendations for the diagnosis and management of PraderWilli syndrome. The Journal of Clinical Endocrinology and Metabolism, 93 (11), 4183 4197. doi:10.1210/jc.2008-0649.

Grolla, E., Andrighetto, G., Parmigiani, P., Hladnik, U., Ferrari, G., Bernardelle, R., Lago, M. D., Albarello, A., Baschirotto, G., Filippi, G., Lovato, R. y Dolcetta, D. (2011). Specific treatment of Prader-Willi syndrome through cyclical rehabilitation programmes. Disability and Rebabilitation, 33 (19-20), 1837-1847. doi:10.3109/09638288.20 10.549288 .

Gunay-Aygun, M., Schwartz, S., Heeger, S., O’Riordan, M. A. y Cassidy, S. B. (2001). The Changing Purpose of Prader-Willi Syndrome Clinical Diagnostic Criteria and Proposed Revised Criteria. Pediatrics, 108 (5), e92-e92. doi:10.1542/peds.108.5.e92.

Jauregi, J., Arias, C., Vegas, O., Alén, F., Martínez, S., Copet, P. y Thuilleaux, D. (2007). A neuropsychological assessment of frontal cognitive functions in Prader-Willi syndrome. Journal of Intellectual Disability Research, 51 (5), 350-365. doi:10.1111/j.13652788.2006.00883.x. 
Liberati, A., Altman, D., Tezzlaff, J., Mulrow, C., Gøtzsche, P., Ioannidis, J., Clarke, M., Devereaux, P. J., Kleijnen, J. y Moher, D. (2009). The PRISMA statement for reporting systematic reviews and meta-analyses of studies that evaluate healthcare interventions: explanation and elaboration. BMJ, 339:b2700. doi: http://dx.doi.org/10.1136/bmj.b2700.

Lo, S. T., Siemensma, E., Collin, P. y Hoknen-Koelega, A. (2013). Impaired theory of mind and symptoms of Autism Spectrum Disorder in children with Prader-Willi syndrome. Research in Developmental Disabilities, 34 (9), 2764-2773. doi:10.1016/j.ridd.2013.05.024.

Lubrini, G., PeriáÑez, J. y Ríos-Lago, M. (2009). Introducción a la estimulación cognitiva y la rehabilitación neuropsicológica. En E. MuÑOZ-MArrón (Ed.), Estimulación cognitiva y rehabilitación neuropsicológica (pp. 13-32). Barcelona: Editorial UOC.

Maglieri, K. A., DeLeon, I. G., Rodriguez-Catter, V. y Sevin, B. M. (2000). Treatment of covert food stealing in an individual with Prader-Willi syndrome. Journal of Applied Behavior Analysis, 33 (4), 615-618. doi:10.1901/jaba.2000.33-615.

Mazaheri, M. M., Rae-Seebach, R. D., Preston, H. E., Schmidt, M., Kountz-Edwards, S., Field, N., Cassidy, S. y Packman, W. (2013). The impact of Prader-Willi syndrome on the family's quality of life and caregiving, and the unaffected siblings' psychosocial adjustment. Journal of Intellectual Disability Research, 57 (9), 861-873. doi:10.1111/j.13652788.2012.01634.x.

McAllister, C. J., Whittington, J. E. y Holland, A. J. (2011). Development of the eating behavior in Prader-Willi Syndrome: advances in our understanding. International Journal of Obesity, 35 (2), 188-197. doi:10.1038/ijo.2010.139.

McCandeess, S. E. y Committee on Genetics (2011). Clinical report-health supervision for children with Prader-Willi syndrome. Pediatrics, 127 (1), 195-204. doi:10.1542/peds.2010-2820.

Messersmith, N. V., Slifer, K. J., Pulbrook-Vetter, V. y Bellipanni, K. (2008). Interdisciplinary Behavioral Intervention for Life-Threatening Obesity in an Adolescent with PraderWilli Syndrome: A Case Report. Journal of Developmental y Behavioral Pediatrics, 29 (2), 129-134. doi:10.1097/DBP.0b013e31815f24bd7.

Misquiatti, A. R., Cristovão, M. P. y Brito, M. C. (2011). Trajectory and outcomes of speech language therapy in the Prader-Willi syndrome (PWS): case report. J Soc Bras Fonoaudiol, 23 (1), 77-81.

Muñoz-MARrón, E. (Ed.) (2009). Estimulación cognitiva y rebabilitación neuropsicológica. Barcelona: Editorial UOC.

Noggle, C., Dean, R. y Barisa, M. (Eds.) (2013). Neuropsychological Rehabilitation. New York, Estados Unidos: Springer Publishing Company.

Pignatti, R., Mori, I., Bertella, L., Grugni, G., Giardino, D. y Molinari, E. (2013). Exploring patterns of unwanted behaviours in adults with Prader-Willi syndrome. J Appl Res Intellect Disabil, 26 (6), 568-577. doi: 10.1111/jar.12047.

Radstaake, M., Didden, R., Bolio, M., Lang, R., Lancioni, G. E. y Curfs, L. M. G. (2011). Functional Assessment and Behavioral Treatment of Skin Picking in a Teenage Girl With Prader-WilliSyndrome. Clinical Case Studies, 10 (1), 67-78. doi:10.1177/1534650110395013.

Rose, J. y Walker, S. (2000). Working with a man who has Prader-Willi syndrome and his support staff using motivational principles. Behavioral and Cognitive Psychotherapy, 28 (3), 293-302.

Samuels, R. y STANSfield, J. (2011). The effectiveness of social stories ${ }^{\mathrm{TM}}$ to develop social interactions with adults with characteristics of autism spectrum disorder. British Journal of Learning Disabilities, 40, 272-285. doi:10.1111/j.1468-3156.2011.00706.x.

Singh, N. N., Lancioni, G. E., Singh, A. N., Winton, A. S. W., Singh, J., McAleavey, K. M. y Adkins, A. D. (2008). A Mindfulness-Based Health Wellness Program for 
an Adolescent with Prader-Willi Syndrome. Behavior Modification, 32 (2), 167-181. doi:10.1177/0145445507308582.

Singh, N. N., Lancioni, G. E., Singh, A. N. A., Winton, A. S. W., Singh, A. D. A. y Singh, J. (2011). A Mindfulness-Based Health Wellness Program for Individuals with Prader-Willi Syndrome. Journal of Mental Health Research in Intellectual Disabilities, 4 (2), 90-106. doi:10.1177/0145445507308582.

Stokes, J. V. y Luiselli, J. K. (2009). Applied Behavior Analysis Assessment and Intervention for Health: Threatening Self-Injury (Rectal Picking) in an Adult with Prader-Willi Syndrome. Clinical Case Studies, 8 (1), 38-47. doi:10.1177/1534650108327011.

Storch, E. A., Rahman, O., Morgan, J., Brauer, L., Miller, J. y Murphy, T. K. (2011). Case Series of Behavioral Psychotherapy for Obsessive-Compulsive Symptoms in Youth with Prader-Willi Syndrome. Journal of Developmental and Physical Disabilities, 23 (4), 359368. doi:10.1007/s10882-011-9233-5.

Van Hooren, R. H., Widdershoven, G. A. M., Candel, M. J. J. M., Van den Borne, B. W. y Curfs, L. M. G. (2006). Between control and freedom in the care for persons with PraderWilli syndrome: an analysis of preferred interventions by caregivers. Patient Education and Counseling, 63 (1-2), 223-231. doi:10.1016/j.pec.2005.11.004.

Whittington, J. (2004). Prader-Willi Syndrome: Development and Manifestations. Cambridge: Cambridge University Press.

Wigren, M. y Hansen, S. (2003). Prader-Willi syndrome: clinical picture, psychosocial support and current management. Child: Care, Health y Development, 29 (6), 449-456. doi:10.1046/ j.1365-2214.2003.00364.x.

Wilson, B., Gracey, F., Evans, J. y Bateman, A. (2009). Neuropsychological Rehabilitation Theory, Models, Therapy and Outcome. Cambridge: Cambridge University Press. 